\title{
BASIC CALCULUS OF VARIATIONS
}

\author{
EDWARD SILVERMAN
}

\begin{abstract}
For the classical one-dimensional problem in the calculus of variations, a necessary condition that the integral be lower semicontinuous is that the integrand be convex as a function of the derivative. We shall see that, if the problem is properly posed, then this condition is also necessary for the $k$-dimensional problem. For the one-dimensional problem this condition is also sufficient. For the $\boldsymbol{k}$-dimensional problem this condition is shown to be sufficient subject to an additional hypothesis. For the one-dimensional problem there is an existence theorem if the integrand grows sufficiently rapidly with respect to the derivative, and this result also holds for the $k$-dimensional problem, subject to an additional hypothesis. Some of these additional hypotheses are automatically satisfied for the one-dimensional problem.
\end{abstract}

Let $G$ be a bounded domain in $\mathbf{R}^{k}, A=G \times \mathbf{R}^{N}, Z$ be the space of $(N \times k)$-matrices and $F \in C(A \times Z)$. If $y: G \rightarrow \mathbf{R}^{N}$ is smooth, let $I_{F}(y)$ $=\int_{G} F\left(x, y(x), y^{\prime}(x)\right) d x$ where $y^{\prime}(x)$ is the matrix of partial derivatives of $y$.

If $k=N=2$ and if $F(a, b, p)=|\operatorname{det} p|$ then $I_{F}$ is the area integral which is lower semicontinuous though $F$ is not convex in $p$ for fixed $(a, b)$. Thus the one-dimensional results do not, apparently, generalize.

There are $r=\left(\begin{array}{c}N+k \\ k\end{array}\right)-1$ Jacobians of orders $1, \ldots, \min \{k, N\}$. Let $Y=\mathbf{R}^{r}$. There exists $\tau: Z \rightarrow Y$ such that $\tau \circ y^{\prime}(x)=J(y, x)$, where $J(y, x)=[J(y)](x)$, and $J(y)$ is the collection of all Jacobians of $y$, whenever $y$ is a smooth map. If $f: A \times Y \rightarrow \mathbf{R}$ and if $f(\theta, \tau(p))=F(\theta, p)$ for all $(\theta, p)$, then, evidently, $I(y)=I_{F}(y)$ where $I(y)=$ $\int_{G} f\left(y_{*}(x), J(y, x)\right) d x$ and $y_{*}(x)=(x, y(x))$.

If $u: V \times W \rightarrow X$ and if $v \in V$ let $u_{v}(w)=u(v, w)$ for each $w \in W$.

We define a class $A C$ of transformations $y$ for which each component of $y$ and each component of $J(y)$, defined in a distribution sense, is in $L=L(G)$. We consider $I(y)$ to be the basic integral, not $I_{F}(y)$.

Let $T=$ range $\tau$. If $k=1$ then $T=Y$ and $T$ can be identified with $Z$ so that $f=F$. In general, however, setting $f_{\theta} \circ \tau=F_{\theta}$ defines $f_{\theta}$ on $T \subset Y$ where $T \neq Y$. Let us say that $f$ is $T$-convex if $f_{\theta}$ can be extended to a function which is convex over all of $Y$ for each $\theta \in A$. Please notice that we do not require that $f_{\theta}$ be convex. What we do require is that there exist a convex function over all of $Y$ which extends $f_{\theta}$. Then a necessary condition that $I$ be lower semicontinuous is that $f$ be $T$-convex. If the extended function is also continuous over $A \times Y$, then the condition is also sufficient.

In some applications $f$, rather than $F$, may be given initially [1]. 
If $k>1$ then the parametric problem is not covered by the existence theorem. Even worse, the dichotomy into parametric and non-parametric problems no longer seems feasible. If $k=N=2$ and if $F(\theta, p)=|\operatorname{det} p|^{2}$ then $I$ is not parametric. Since it is invariant under smooth area-preserving changes of variables, it has something of the distinguishing feature of parametric integrals. Here $r=5$ and $f_{\theta}(t)$ depends upon a single component of $t$. Thus $f_{\theta}$ does not grow with $\|t\|$.

The starting point of this paper is [5]. Morrey's sufficiency condition for quasiconvexity gave the idea of using $f$ rather than $F$. That idea, together with the notion of the Cesari-Weierstrass integral [2] and the ideas used in [7] and [8] led to the sufficient condition. The compactness results are familiar [6]. The consistent use of quasilinear functions to approximate continuous functions, rather than Lipschitzian or smoother functions, is standard in area theory, especially in Cesari's papers.

2. If $y$ is smooth then each component of $J(y)$ is the determinant of a submatrix of order $k$ of $y_{*}^{\prime}$, except possibly for sign. One of these submatrices is the identity. Its determinant does not correspond to any component of $J(y)$. Thus $J(y)$ has $r$ components. Let $Y=\mathbf{R}^{r}$.

If $M \geq m$ let $\Lambda(M, m)$ be the collection of all strictly increasing $m$-termed sequences taken from $\{1, \ldots, M\}$. Let $s=\min \{k, N\}$. If $j \leq s$, if $i \in \Lambda(N, j)$ and $\alpha \in \Lambda(k, j)$, let $p_{\alpha}^{i}=\operatorname{det}\left[\left(p_{\alpha_{m}}\right)^{i_{n}}\right]_{1 \leq m, n \leq 1}$ and define $\tau: Z \rightarrow Y$ by $\tau(p)=\left\{p_{\alpha}^{i} \mid(i, \alpha) \in \cup_{j=1}^{s}(\Lambda(N, j) \times \Lambda(k, j))\right\}$. We may write $\left.{ }_{p}^{I}\right]$ for $\tau(p)$. Similarly, if $\phi$ is a $(k \times k)$-matrix then the determinants of the $(k \times k)$-submatrices of $\left[\begin{array}{l}\phi \\ p\end{array}\right]$ are in 1-1 correspondence with those of $\left[{ }_{p}^{I}\right]$. (We delete the determinant of the top matrix, of course.)

Evidently there exists a unique linear map $\phi: Y \rightarrow Z$ such that $\Psi \circ \tau(p)=p$ for each $p \in Z$.

If $(i, \alpha) \in \cup_{j=1}^{s}(\Lambda(M, j) \times \Lambda(k, j))$ then there exists $\lambda, 1 \leq \lambda \leq r$, such that

$$
\frac{\partial\left(y^{i_{1}}, \ldots, y^{i_{\jmath}}\right)}{\partial\left(x^{\alpha_{1}}, \ldots, x^{\alpha_{\jmath}}\right)}=\frac{d y^{i}}{d x^{\alpha}}= \pm \tau\left(y^{\prime}\right)^{\lambda}
$$

We can suppose that, if $N \geq k$ and $j=s=k$, then $r_{0}=\left(\begin{array}{c}N+k \\ k\end{array}\right)-\left(\begin{array}{c}N \\ k\end{array}\right) \leq$ $\lambda \leq r$.

The components of $J(y)$ are, except possibly for sign, the components of $\tau\left(y^{\prime}\right)$. Thus there is no loss in generality in ordering the rows of the submatrices in such a way that we can identify $J(y)$ with $\tau\left(y^{\prime}\right)$.

3. To obtain the necessary condition for lower semicontinuity we require some information about $\tau$. 
LEMMA 3.1. Let $\mu_{n} \in \mathbf{R}, n=1, \ldots, m$, with $\Sigma \mu_{n}=1$. If $p_{n}, p$ and $q \in Z$ with $\Sigma \mu_{n} \tau\left(p_{n}\right)=\tau(p)$ then $\Sigma \mu_{n}\left(p_{n}+q\right)^{1} \wedge \cdots \wedge\left(p_{n}+q\right)^{j}=$ $(p+q)^{1} \wedge \cdots \wedge(p+q)^{j}$ for $j=1, \ldots, k$.

Proof. We expand and get $(p+q)^{1} \wedge \cdots \wedge(p+q)^{j}=$ $p^{1} \wedge \cdots \wedge p^{j}+\sum_{i=1}^{j-1} \Sigma^{\prime} \varepsilon_{\alpha, i} p^{\alpha_{1}} \wedge \cdots \wedge p^{\alpha_{1}} q^{\gamma_{1}} \wedge \cdots \wedge q^{\gamma_{j-1}}+q^{1} \wedge \cdots \wedge q^{j}$ where $\Sigma^{\prime}$ is the sum over $\alpha \in \Lambda(j, i)$ and $\gamma \in(1, \ldots, j) \sim\{\alpha\}$. Also, $\varepsilon_{\alpha, i}= \pm 1$. Then

$$
\begin{aligned}
\sum \mu_{n}\left(p_{n}+q\right)^{1} \wedge \cdots \wedge\left(p_{n}+q\right)^{j} & \\
= & p^{1} \wedge \cdots \wedge p^{j}+\sum_{n=1}^{m} \mu_{n} \sum_{i=1}^{j-1} \sum^{\prime} \varepsilon_{\alpha, i} p_{n}^{\alpha_{1}} \wedge \cdots \wedge p_{n}^{\alpha_{i}} \wedge q^{\gamma_{1}} \wedge \cdots \wedge q^{\gamma_{j-i}} \\
& \quad+q^{1} \wedge \cdots \wedge q^{j}=(p+q)^{1} \wedge \cdots \wedge(p+q)^{j} .
\end{aligned}
$$

Corollary 3.2. $\tau(p+q)=\Sigma \mu_{n} \tau\left(p_{n}+q\right)$.

LEMMA 3.3. Let $y: \mathbf{R}^{k} \rightarrow \mathbf{R}^{N}$ be quasilinear with compact support $K$ and simplexes of linearity $\delta_{1}, \ldots, \delta_{m}$. Let $p_{n}=y^{\prime}(x)$ for $x \in \operatorname{Int} \delta_{n}$ and let $\mu_{n}=\left|\delta_{n}\right| /|K|$. Then $\mu_{n}>0, \Sigma \mu_{n}=1$ and $\Sigma \mu_{n} \tau\left(p_{n}\right)=0$.

Except for notation, this is Lemma 4.4 [6].

It is not hard to verify that $Y$ is the convex hull of $T$.

Let us say that $I$ is 1 sc if $I(y) \leq \liminf I\left(y_{n}\right)$ whenever $y_{n}$ converges uniformly to $y, y_{n}$ and $y$ satisfy a uniform Lipschitz condition (which may depend upon the sequence) and $y_{n}-y$ is quasilinear with support contained in a cube contained in $G$. (See Def. 4.4.2, [6].)

If $N \geq k$ and if $f(\theta, q)=f\left(\theta,\left(0, \ldots, 0, q^{r_{0}}, \ldots, q^{r}\right)\right)$ for each $\theta \in A$ then we say that $f$ depends only upon Jacobians of maximum rank.

LEMMA 3.4. Let $f$ depend only upon Jacobians of maximum rank and suppose that $f_{\theta} \in C^{\prime}$ for each $\theta \in A$. If I is lsc then then $f$ is $T$-convex.

Proof. If $f_{\theta}(\tau(p)) \leq \Sigma \lambda_{\beta} f_{\theta}\left(\tau\left(p_{\beta}\right)\right)$ whenever $\theta \in A, p, p_{\beta} \in Z, \lambda_{\beta}>0$, $\Sigma \lambda_{\beta}=1$ and $\Sigma \lambda_{\beta} \tau\left(p_{\beta}\right)=\tau(p)$, then $t \mapsto \inf \left\{\Sigma \lambda_{\beta} \tau\left(p_{\beta}\right) \mid \Sigma \lambda_{\beta} \tau\left(p_{\beta}\right)=t\right\}$ is an extension of the required type. If

$$
f_{\theta}(\tau(q)) \geq f_{\theta}(\tau(p))+f_{\theta}^{\prime}(\tau(p)) \tau(q-p)
$$

for all $\theta \in A, p$ and $q \in Z$, then by Corollary 3.2, $\Sigma \lambda_{\beta} \tau\left(p_{\beta}-p\right)=\tau(0)$ so $\Sigma \lambda_{\beta} f_{\theta}\left(\tau\left(p_{\beta}\right)\right) \geq \Sigma \lambda_{\beta} f_{\theta}(\tau(p))+f_{\theta}^{\prime}(\tau(p)) \Sigma \lambda_{\beta} \tau\left(p_{\beta}-p\right)=\Sigma \lambda_{\beta} f_{\theta}(\tau(p))=$ $f_{\theta}(\tau(p))$. 
Let $Q=\mathbf{R}^{k} \cap\left\{x \mid-\frac{1}{2} \leq x^{1}, \ldots, x^{k} \leq \frac{1}{2}\right\}$ and let $h>0$. Let $p \in Z$. Then $Q$ is partitioned into $3^{k}$ cells by the hyperplanes $x^{\alpha}= \pm h / 2$, $\alpha=1, \ldots, k$. Each of these cells, except $h Q$, is then subdivided into $k$ ! simplexes whose vertices are contained in the set of vertices of the containing cell. Let $S$ be the set of all these simplexes. Now we define $\alpha$ continuous (quasilinear) function $\zeta$ on $Q$ into $\mathbf{R}^{N}$ by putting $\zeta(x)=p x$ if $x \in h Q, \zeta(x)=0$ if $x \in \partial Q$ and $\zeta \mid \sigma$ is linear (affine) if $\sigma \in S$. If $x \in$ Int $\sigma$ let $\zeta^{\prime}(x)=p_{\sigma}$. Thus, by Lemma 3.3, $\tau(p) h^{k}+\Sigma_{\sigma \in S} \tau\left(p_{\sigma}\right)|\sigma|=$ 0 . Also, for each $\sigma \in S$ there exists $j \in\{1, \ldots, k\}$ such that $j$ columns of $p_{\sigma}$ are $O(h)$ and $|\sigma|=O\left(h^{k-j}\right)$. By Theorem 4.4.2 [6],

$$
\begin{aligned}
f_{\theta}(\tau(0)) \leq & \int_{Q} f_{\theta}\left(\tau\left(\zeta^{\prime}(x)\right)\right) d x=f_{\theta}(\tau(p)) h^{k}+\sum_{\sigma \in s} f_{\theta}\left(\tau\left(p_{\sigma}\right)\right)|\sigma| \\
= & f_{\theta}(\tau(p)) h^{k}+\sum_{\sigma \in s}\left[f_{\theta}(\tau(0))+f_{\theta}^{\prime}(\tau(0)) \tau\left(p_{\sigma}\right)+o\left(\tau\left(p_{\sigma}\right)\right)\right]|\sigma| \\
= & f_{\theta}(\tau(p)) h^{k}+f_{\theta}(\tau(0))\left(1-h^{k}\right)-f_{\theta}^{\prime}(\tau(0)) \tau(p) h^{k} \\
& +\sum_{\sigma \in s} O\left(\tau\left(p_{\sigma}\right)\right)|\sigma|
\end{aligned}
$$

so that $f_{\theta}(\tau(0)) h^{k}+f_{\theta}^{\prime}(\tau(0)) \tau(p) h^{k} \leq f_{\theta}(\tau(p)) h^{k}+\Sigma_{\sigma \in S} O\left(\tau\left(p_{\sigma}\right)\right)|\sigma|$. If $f$ depends only upon Jacobians of rank $k$, then the last term on the right is $o\left(O\left(h^{k}\right)\right)=o\left(h^{k}\right)$ so that $f_{\theta}(\tau(p)) \geq f_{\theta}(\tau(0))+f_{\theta}^{\prime}(\tau(0)) \tau(p)$.

COROllary 3.5. The lemma remains valid if the differentiability condition is dropped.

Proof. Let $F_{\theta}=f_{\theta} \circ \tau$ and suppose that $F_{\theta} \in C^{\prime}$. Then $f_{\theta}=F_{\theta} \circ \Psi$, $f_{\theta}^{\prime}=\left(F_{\theta}^{\prime} \circ \Psi\right) \Psi^{\prime}$ and $f_{\theta} \in C^{\prime}$. If $F_{\theta} \notin C^{\prime}$ we mollify. Let $B$ be the unit sphere in $Z$, let $\mu \in C^{\infty}(Z)$ be nonnegative with support contained in $B$ and $\int \mu(\xi) d \xi=1$. If $\rho>0$ let $\mu_{\rho}(\xi)=1 / \rho^{N k} \mu(\xi / p)$.

If $y_{n} \rightarrow y$ then $y_{n}-\xi \rightarrow y-\xi$ where, because of the definition of 1sc, we can suppose that $y_{n}-\xi$ and $y-\xi$ differ only on a compact subset of $G$. A routine argument shows that $y \mapsto \int_{G} F\left(y_{*}(x), y^{\prime}(x)-\xi\right) d x$ is lsc. Thus

$$
y \mapsto \int_{R} F_{\rho}\left(y_{*}(x), y^{\prime}(x)\right) d x
$$

is 1sc where $F_{\rho}(\theta, p)=\int_{\rho B} F\left((\theta, p-\xi) \mu_{\rho} \mid \xi\right) d \xi$. Let $f_{\rho}(\theta, q)=F_{\rho}(\theta, \Psi q)$. Then $\left(f_{\rho}\right)_{\theta} \in C^{\prime}$ since $\left(F_{\rho}\right)_{\theta} \in C^{\prime}$. Thus, by the lemma, $f_{\rho}$ is $T$-convex and the corollary follows by letting $\rho \rightarrow 0$.

THEOREM 3.6. Let I be lsc. Then $f$ is T-convex. 
Proof. If $\theta \in A$ let $g\left(\theta,\left[\begin{array}{l}\phi \\ ]\end{array}\right)=g_{\theta}\left(\left[{ }_{p}^{\phi}\right]\right)=f_{\theta}\left(\left[\begin{array}{l}I \\ p\end{array}\right]\right)\right.$. (See §2.) Now let

$$
h\left(\theta,\left[\begin{array}{l}
I \\
\phi \\
p
\end{array}\right]\right)=g\left(\theta,\left[\begin{array}{l}
\phi \\
p
\end{array}\right]\right) .
$$

Let $Z_{0}, Y_{0}$ and $\Psi_{0}$ correspond to $Z, Y$ and $\Psi$ with $\mathbf{R}^{N+k}$ replacing $\mathbf{R}^{N}$. Let $h_{\theta}$ be defined over all of $Y_{0}$ by $h_{\theta}(q)=h_{\theta}(r)$ if $\Psi_{0} q=\Psi_{0} r$. By this construction $h \in C\left(A \times Y_{0}\right), h$ is nonnegative and $h$ depends only upon Jacobians of maximum rank.

If $(\xi, y): G \rightarrow \mathbf{R}^{k} \times \mathbf{R}^{N}$ then let

$$
\begin{aligned}
I_{h}(\xi, y) & =\int_{G} h\left(y_{*}(x),\left[\begin{array}{c}
I \\
\xi^{\prime}(x) \\
y^{\prime}(x)
\end{array}\right]\right) d x \\
& =\int_{G} g\left(y_{*}(x),\left[\begin{array}{c}
\xi^{\prime}(x) \\
y^{\prime}(x)
\end{array}\right]\right) d x=I(y)
\end{aligned}
$$

and $I_{h}$ is lsc. Thus $h$ is $T$-convex. In a natural way $Y=\operatorname{dom} f_{\theta} \subset \operatorname{dom} h_{\theta}$. Furthermore, $h_{\theta}$ extends $f_{\theta} \mid T$. Thus $g_{\theta}=h_{\theta} \mid Y$ is an extension of $f_{\theta} \mid T$ which is convex over all of $Y$.

4. In this section we define a class of transformations, which we call $A C$, on which $I$ is defined. This class is probably not a vector space.

Let $\mathscr{Q}=C_{o}^{\infty}(G), L=L_{1}(G)$ and $L_{p}=L_{p}(G)$ for $p>1$. If $B$ is one of these spaces let $F_{0} B=B, F_{j} B=0$ if $j>k$ and, if $1 \leq j \leq k$, let

$$
F_{j} B=\left\{\omega \mid \omega=\sum_{\lambda \in \Lambda(k, j)} \omega_{\lambda} d x^{\lambda} \text { where each } \omega_{\lambda} \in B\right\} .
$$

As usual, $d x^{\lambda}=d x^{\lambda_{1}} \wedge \cdots \wedge d x^{\lambda_{J}}$.

If $\omega \in F_{j} L$ and if there exists $\zeta \in F_{j+1} L$ such that

$$
\int \omega \wedge d \phi=(-1)^{j+1} \int \zeta \wedge \phi
$$

for each $\phi \in F_{k-j-1} \mathscr{Q}$, then we say that $\omega \in \mathscr{F}_{j} H$ and write $d \omega$ for $\zeta$. If $d \omega$ exists, then $d \omega$ is unique.

By putting an appropriate norm on $\mathscr{F}_{o} H$ we can identify this space with $H=H_{1}^{1}(G)$. Also, $H_{o}=H_{1, o}^{1}(G)$ is the closure, in $H$, of $\mathscr{D}=\mathscr{F}_{o} \mathscr{D}$.

If $\omega_{n}=\Sigma \omega_{n \lambda} d x^{\lambda}$ and $\omega=\sum \omega_{\lambda} d x^{\lambda}$ are in $F_{j} L$ then $\omega_{n}-\omega$ in $F_{j} L$ if $\omega_{n \lambda} \rightarrow \omega_{\lambda}$ in $L$ for each $\lambda$, where $\rightarrow$ denotes weak convergence on compact subsets of $G$.

LEMMA 4.1. If $\omega_{n} \rightarrow \omega$ in $F_{j} L$, if $\omega_{n} \in \mathscr{F}_{j} H$ and if $d \omega_{n} \rightarrow \zeta$ in $F_{j+1} L$ then $\omega \in \mathscr{F}_{j} H$ and $d \omega=\zeta$. 
Proof. Let $\phi \in F_{k-j-1}$ 冈. Then

$$
\int \omega \wedge d \phi=\lim \int \omega_{n} \wedge d \phi=(-1)^{j+1} \lim \int d \omega_{n} \wedge \phi=(-1)^{j+1} \int \zeta \wedge \phi
$$

LEMMA 4.2. If $\omega \in \mathscr{F}_{j} H$ then $x^{\alpha} \omega \in \mathscr{F}_{j} H$ and

$$
d\left(x^{\alpha} \omega\right)=d x^{\alpha} \wedge \omega+x^{\alpha} d \omega .
$$

Proof. Let $\phi \in F_{k-j-1} \mathscr{D}$ and $\psi=x^{\alpha} \phi$ so that $d \psi=d x^{\alpha} \wedge \phi+x^{\alpha} d \phi$ and

$$
\begin{aligned}
\int x^{\alpha} \omega \wedge d \phi & =\int \omega \wedge\left[d \psi-d x^{\alpha} \wedge \phi\right] \\
& =\int \omega \wedge d \psi+(-1)^{j+1} \int d x^{\alpha} \wedge \omega \wedge \phi \\
& =(-1)^{j+1} \int\left(x^{\alpha} d \omega+d x^{\alpha} \wedge \omega\right) \wedge \phi
\end{aligned}
$$

LeMma 4.3. If $\omega \in \mathscr{F}_{j} H$ then $d^{2} \omega=0$.

Proof. Let $\zeta=d \omega$ and $\phi \in F_{k-j-2} \mathscr{D}$. Then $\int \zeta \wedge d \phi=(-1)^{j} \int \omega \wedge d^{2} \phi$ $=0=(-1)^{j} \int 0$ so that $d^{2} \omega=d \zeta=0$.

If $z \in H$ then $d z=\Sigma_{\alpha \in \Lambda(k, 1)} z_{\alpha} d x^{\alpha}$ where $\left\{z_{\alpha}\right\}$ is the set of distribution derivatives of $z$. Let $M$ be a positive integer and $s=\min \{k, M\}$. Suppose that $d z^{i}$ has been defined for $i \in \Lambda(M, j), j \leq s-1$. If $h \in$ $\Lambda(M, j+1), m=h_{1}$ and $i=h \sim\{m\} \in \Lambda(M, j)$ then we define $d z^{h}$, if $z^{m} d z^{i} \in \mathscr{F}_{j} H$, by $d z^{h}=d\left(z^{m} d z^{i}\right)$. by

If $d z^{i}$ is defined for $i \in \Lambda(M, j)$ and $\alpha \in \Lambda(k, j)$ then we define $z_{\alpha}^{i}$

$$
d z^{i}=\sum_{\alpha \in \Lambda(k, j)} z_{\alpha}^{i} d x^{\alpha}
$$

so that, if $z$ is smooth, $z_{\alpha}^{i}=\left(\partial\left(z^{i_{1}}, \ldots, z^{i_{j}}\right) / \partial\left(x^{\alpha_{1}}, \ldots, x^{\alpha_{j}}\right)\right)$.

Let $y \in L^{N}$ and suppose that $d y^{i}$ is defined for each $i \in \Lambda(M, s)$, where $s=\min \{N, k\}$, and thus for each $i \in \bigcup_{j=1}^{s} \Lambda(M, j)$. Then we can suppose that $J(y)=\left\{y_{\alpha}^{i} \mid(i, \alpha) \in \bigcup_{j=1}^{s}(\Lambda(N, j) \times \Lambda(k, j))\right\}$ is an element of $L^{r}$.

If $J(y)$ is defined and if $J(y)=\tau\left(y^{\prime}\right)$ almost everywhere then we say that $y \in A C$. By the definition of $\mathscr{F}_{j} H$, the components of $J(y)$ are functions.

The following lemmas are immediate. 
LEMma 4.4. $y_{*} \in A C$ if and only if $y \in A C$ and $J(y)=\left\{y_{* \beta}^{i} \mid i \in\right.$ $\Lambda(k+N, j)$ and $\beta=(1, \ldots, k)\}$.

LEMma 4.5. Let $j \leq s=\min \{N, k\}$ and $y \in A C$. If $(i, \alpha) \in$ $\Lambda(N, j) \times \Lambda(k, j)$ for $1 \leq j \leq s$ then there exists $h \in \Lambda(k+N, k)$ such that, except possibly for sign, $y_{* \beta}^{h}=y_{\alpha}^{i}$.

Let $y_{n} \in A C$ and $y \in L^{N}$ with $y_{n *}^{m} \rightarrow y_{*}^{m}$ in $L$ for each $m \in$ $\Lambda(k+N, 1)$. Suppose that if $j \leq k$ and $i \in \Lambda(k+N, j)$ there exists $\zeta^{i} \in F_{j} L$ such that $d y_{n *}^{i}-\zeta^{i}$ in $F_{j} L$. If, in addition, $y_{n *}^{m} d y_{n *}^{i} \rightarrow y_{*}^{m} \xi^{i}$ in $F_{j} L$ whenever $i \in \Lambda(k+N, j), j<k, m \in \Lambda(k+N, 1)$, and $m \notin i$ then we say that $y_{n} \Rightarrow y$.

THEOREM 4.6. If $y_{n} \Rightarrow y$ then $y \in A C$ and $J\left(y_{n}\right) \rightarrow J(y)$ in $L$.

Proof. By Lemma 4.1, $J(y)$ is defined. By Theorem 3.4 .4 [6], $y_{n *}^{m} d y_{n *}^{i}-y_{*}^{m} d y_{*}^{i}$ in $L(K)$ for each compact set $K \subset G$. Hence we can suppose that $y_{n *}^{m} d y_{n *}^{i} \rightarrow y_{*}^{m} d y_{*}^{i}$ almost everywhere in $G$. We can also suppose that $i \neq(1,2, \ldots, k)$. Hence there exists $m \in\{1, \ldots, k\}, m \notin i$, such that $x^{m} d y_{n *}^{i} \rightarrow x^{m} d y_{*}^{i}$ so that $d y_{n *}^{i} \rightarrow d y_{*}^{i}$ almost everywhere.

LEMMA 4.7. If $p$ and $q$ are Lebesgue conjugate, if $f_{n} \rightarrow f$ in $L_{p}$ and $g_{n} \rightarrow g$ in $L_{q}$ then $f_{n} g_{n} \rightarrow f g$ in $L$.

Proof. Let $E$ be a measurable subset of a compact subset of $G$. Then

$$
\int_{E}\left(f_{n} g_{n}-f_{g}\right) d x=A_{n}+B_{n}
$$

where $A_{n}=\int_{E} f\left(g_{n}-g\right) d x$ and $B_{n}=\int_{E}\left(f_{n}-f\right) g_{n} d x$. By the weak convergence, $A_{n} \rightarrow 0$ and $\left\{\int_{E}\left|g_{n}(x)\right|^{q} d x\right\}^{1 / q}$ is bounded independently of $n$. Thus $B_{n} \rightarrow 0$ by the Hölder inequality.

If $y \in A C$ and if $y_{* \beta}^{i} \in L_{p}$ for each $i \in \Lambda(k \times N, k)$, where $\beta=$ $(1, \ldots, k)$, then we set $\|J(y)\|_{p} \stackrel{p}{=} \Sigma_{i \in \Lambda(k \times N, k)}\left\|y_{* \beta}^{i}\right\|_{p}$.

If $y_{o} \in A C$ let $\Re\left(y_{o}\right)=A C \cap\left\{y \mid y-y_{o} \in\left(H_{o}\right)^{N}\right\}$.

THEOREM 4.8. Suppose that there exists $M>0$ such that for each $y \in \mathfrak{N}\left(y_{o}\right)$ either

(i) $\|y\|_{\infty} \leq M$ and $\|J(y)\|_{p} \leq M$ for some $p>1$, or

(ii) $\|J(y)\|_{q} \leq M$ where $q=2 k /(k+1)$. Then $\mathfrak{N}\left(y_{o}\right)$ is $\Rightarrow$ sequentially compact.

Proof. If (i) holds then $\|y\|_{1}^{1}$ is uniformly bounded so that there exists a sequence $\left\{y_{n}\right\}$ in $\Re\left(y_{o}\right)$ and $\zeta \in\left(H_{o}\right)^{N}$ such that $y_{n}-y_{o}-\zeta$ in $\left(H_{o}\right)^{N}$. 
Thus $y_{n}-y_{o} \rightarrow \zeta$ in $L$. Let $y=y_{o}+\zeta$. By passing to a subsequence we can suppose that $y_{n}(x) \rightarrow y(x)$ a.e. By the bounded convergence theorem, $y_{n *} \rightarrow y_{*}$ in $\left(L_{s}\right)^{N}$ where $s=p /(p-1)$ is Lebesgue conjugate to $p$. If (ii) holds then there exists a sequence $\left\{y_{n}\right\}$ in $\Re\left(y_{o}\right)$ and $\zeta \in\left(H_{q, o}\right)^{N}$ such that $y_{n}-y_{o}-\zeta$ in $\left(H_{p, o}\right)^{N}$. Thus, by Th. 3.5.3, [6], $y_{n} \rightarrow y$ in $L_{t}$ where $1 / t=1 / q-1 / k=(k-1) / 2 k$ so that $t$ is conjugate to $q$. The theorem follows by induction, Lemma 4.1 and Lemma 4.7.

5. We make use of a type of convexity studied by Tonelli to show that $T$-convexity is sufficient for lower semicontinuity.

According to Tonelli, a $T$-convex function $f$ is semi-regular positive semi-normal if for each $\theta \in A, p, q \in Y$ with $q \neq 0$, there exists $\lambda \in \mathbf{R}$ such that $2 f(\theta, p)<f(\theta, p+\lambda q)+f(\theta, p-\lambda q)$.

For the following lemma see Turner [10].

LEMMA 5.1. A necessary and sufficient condition that $f$ be semi-regular positive semi-normal is that for each $\varepsilon>0$ and each $(\theta, p) \in A \times Y$, there exists $\delta>0, \nu>0, \zeta \in Y^{*}$ and $\rho \in \mathbf{R}$ such that for all $\phi \in A$ with $\|\phi-\theta\|<\delta$,

(a) $f(\phi, q) \geq \zeta q+\rho+\nu\|q-p\|$ for each $q \in Y$ and

(b) $f(\phi, q) \leq \zeta q+\rho+\varepsilon$ if $\|q-p\|<\delta$.

Let $f$ be semi-regular positive. If $\zeta \in Y^{*}$ let

$$
\rho_{\zeta}(\theta)=\inf \{f(\theta, q)-\zeta q \mid q \in Y\}
$$

for each $\theta \in A$. Thus $f(\theta, p)=\sup \left\{\zeta p+\rho_{\zeta}(\theta) \mid \zeta \in Y^{*}\right\}$.

Let $\sigma_{\zeta}(\phi)=\liminf _{\theta \rightarrow \phi} \rho_{\zeta}(\theta)$ where $\theta$ and $\phi$ belong to $A$, of course. Then $\rho_{\zeta}$ is upper semicontinuous, $\sigma_{\zeta}$ is lower semicontinuous and $\sigma_{\zeta} \leq \rho_{\zeta}$.

THEOREM 5.2. If $f$ is semi-regular positive semi-normal, then $f(\theta, p)=$ $\sup \left\{\zeta p+\sigma_{\zeta}(\theta) \mid \zeta \in Y^{*}\right\}$.

Proof. Let $\varepsilon>0$. By Lemma 5.1 there exist $\delta>0, \nu>0, \zeta \in Y^{*}$ and $\rho \in \mathbf{R}$ such that if $\phi \in A$ and $\|\phi-\theta\|<\delta$, then

(a) $f(\phi, q) \geq \zeta q+\rho+\|q-p\|$ for each $q \in Y$, and

(b) $f(\phi, q) \leq \zeta q+\rho+\varepsilon$ if $\|q-p\|<\delta$.

Hence $\rho_{\zeta}(\phi) \geq \rho$ for each $\phi \in A$ with $\|\phi-\theta\|<\delta$ so that $\sigma_{\xi}(\theta) \geq \rho$ and $f(\theta, p) \leq \zeta p+\sigma_{\zeta}(\theta)+\varepsilon$.

We say that $f$ is $V$-convex if $f(\theta, p)=\sup \left\{\zeta p+\sigma_{\zeta}(\theta) \mid \zeta \in Y^{*}\right\}$ for each $\theta \in A$. Thus $f$ is $V$-convex if $f$ is semi-regular positive semi-normal.

6. In this section we show that if $f \in C(A \times Y)$ is nonnegative and $T$-convex, then $I$ is lower semicontinuous. 
Let $\left\{e^{\lambda}\right\}$ be a dual basis for $Y^{*}=e^{\lambda} e_{\mu}=\delta_{\mu}^{\lambda}$ for $e_{\mu} \in Y$. If $\zeta \in Y^{*}$ there exist $\zeta_{\lambda} \in \mathbf{R}$ such that $\zeta=\Sigma \zeta_{\lambda} e^{\lambda}$.

Let $\delta$ be the collection of all finite families $\sigma$ of compact subsets contained in $G$ such that if $K \in \sigma$ and $L \in \sigma,|K \cap L|=0$ whenever $K \neq L$.

If $y \in A C, \zeta \in Y^{*}$ and $K$ is a compact subset of $G$, let $A(\zeta, y, K)=$ $\zeta\left(\int_{K} J(y, x) d x\right)=\int_{K} \zeta(J(y, x)) d x$ and

$$
B(\zeta, y, K)=\left(\inf \left\{\sigma_{\zeta}\left(y_{*}(x)\right)\right\} \mid x \in K\right)|K| .
$$

Now we define 9 on $A C$ by

$$
g(y)=\sup _{\sigma \in S} \sum_{K \in \sigma} \sup _{\zeta \in Y^{*}}[A(\zeta, y, K)+B(\zeta, y, K)] .
$$

Lemma 6.1. Let $y_{n}$ and $y_{o}$ belong to AC with $y_{n}-y_{o} \in\left(H_{o}\right)^{N}$. If $y_{n}-y_{o} \rightarrow \zeta$ in $H^{N}$ and if we set $y=y_{o}+\zeta$ then $y-y_{o} \in\left(H_{o}\right)^{N}$ and $y_{n} \rightarrow y$ in $\left(L_{1}(K)\right)^{N}$ for each compact subset $K$ of $G$.

This lemma follows from Theorems 3.2.1 and 3.4.4 [6].

LEMMA 6.2. Let $X$ be a measurable subset of $G$ and $\left\{f_{n}\right\}$ be a sequence of measurable functions with $f_{n}(x) \rightarrow f(x)$ a.e. in $X$. Let $\varepsilon>0$. Then there exists a compact set $K \subset X$ with $|X \sim K|<\varepsilon, f_{n} \mid K$ continuous for each $n$ and $f_{n}|K \rightarrow f| K$ uniformly.

This lemma follows from Egoroff's Theorem and Lusin's Theorem.

THEOREM 6.3. Let $f$ be $V$-convex and suppose that $y_{n}$ and $y$ are in

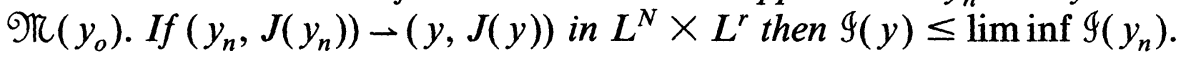

Proof. Let $K$ be a compact subset of $G$. By Lemma 6.1 we can suppose that $y_{n} \rightarrow y$ in $L(K)^{N}$ so that (passing to a subsequence if necessary) $y_{n}(x) \rightarrow y(x)$ for almost all $x \in K$. Let $M>0, \sigma_{\zeta}^{M}(\theta)=$ $\min \left\{\sigma_{\zeta}(\theta), M\right\}$ and let $f^{M}(\theta, p)=\sup \left\{\zeta p+\sigma_{\zeta}^{M}(\theta) \mid \zeta \in Y^{*}\right\}$. It is sufficient to show that the theorem holds with $f$ replaced by $f^{M}$. Hence we can suppose that $\sigma_{\zeta}(\theta) \leq M$ for all $(\theta, \zeta) \in A \times Y^{*}$. Let $\varepsilon>0$. There exists $\eta \in(0, \varepsilon / M)$ such that $\int_{E} \zeta\left(J\left(y_{*}(x)\right)\right) d x<\varepsilon$ if $E$ is a measurable subset of $K$ with $|E|<\eta$. By Lemma 6.2 there exists a compact set $C \subset K$ such that $|K \sim C|<\eta, y_{n} \mid C$ is continuous and $y_{n} \rightarrow y$ uniformly on $C$. Hence

$$
\begin{aligned}
B(\zeta, y, C) & =\left(\inf _{x \in C} \sigma_{\zeta}\left(y_{*}(x)\right)\right)|C| \\
& \geq\left(\inf _{x \in K} \sigma_{\zeta}\left(y_{*}(x)\right)\right)|C| \geq B(\zeta, y, K)-\varepsilon .
\end{aligned}
$$


Also there exist $x_{n} \in C$ such that $\sigma_{\zeta}\left(y_{n *}\left(x_{n}\right)\right)=\inf _{x \in C} \sigma_{\zeta}\left(y_{n *}(x)\right)$. We can suppose that $x_{n} \rightarrow x \in C$. Now $y_{n}\left(x_{n}\right) \rightarrow y(x)$ so that $\sigma_{\zeta}\left(y_{*}(x)\right) \leq$ $\lim \inf \sigma_{\zeta}\left(y_{n *}\left(x_{n}\right)\right)$. Thus $B(\zeta, y, C) \leq \lim \inf B\left(\zeta, y_{n}, C\right)$ while $A(\zeta, y, C)=\lim A\left(\zeta, y_{n}, C\right)$. The theorem follows.

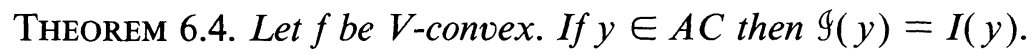

Proof. Let $K$ be a compact subset of $G$ and $\zeta \in Y^{*}$. Then

$$
\begin{aligned}
& \int_{K} f\left(y_{*}(x),\right.J(y, x)) d x \\
& \geq \int_{K}\left[\zeta(J(y, x))+\sigma_{\zeta}\left(y_{*}(x)\right)\right] d x \geq A(\zeta, y, K)+B(\zeta, y, K)
\end{aligned}
$$

so that $I(y) \geq \mathscr{G}(y)$ and we can suppose that $\Phi(y)<\infty$. If $L$ is an interval contained in $G$ let $\mathcal{S}_{L}=\mathcal{S} \cap\left\{\sigma \mid \cup_{K \in \sigma} K \subset L\right\}$ and let

$$
\Phi(L)=\sup _{\sigma \in \delta_{L}} \sum_{K \in \sigma} \sup _{\zeta \in Y^{*}}[A(\zeta, y, K)+B(\zeta, y, K)] .
$$

Then $\Phi$ is nonnegative, superadditive and of bounded variation. Let $D \Phi$ be the Lebesgue derivative of $\Phi$ with respect to cubes. Then $D \Phi(x) \geq$ $\zeta(J(y, x))+\sigma_{\xi}\left(y_{*}(x)\right)$ so that $D \Phi(x) \geq f\left(y_{*}(x), J(y, x)\right)$ almost everywhere in $G$. Evidently $\Phi(y) \geq \sup _{\sigma \in \mathcal{S}^{\prime}} \Sigma_{L \in \sigma} \Phi(E)$ where $\mathcal{S}^{\prime}=\mathcal{S} \cap\{\sigma \mid \sigma$ is a family of finitely many non-overlapping intervals $\}$. Thus $g(y) \geq$ $\sup _{\boldsymbol{\sigma} \in \mathcal{S}^{\prime}} \Sigma_{L \in \boldsymbol{\sigma}} \int_{L} f\left(y_{*}(x), J(y, x)\right) d x=I(y)$.

Corollary 6.5. The theorem holds if $f \in C(A \times Y)$ and $f_{\theta}$ is convex for each $\theta \in A$. Thus $I$ is lsc if $f$ is continuous and T-convex.

Proof. Let $\varepsilon>0$ and $g(\theta, q)=f(\theta, q)+\varepsilon\|q\|$ for each $(\theta, q) \in$ $A \times Y$. Let $I_{g}(y)=\int_{G} g\left(y_{*}(x), J(y, x)\right) d x$. If $J\left(y_{n}\right) \rightarrow J(y)$ in $L^{r}$ then there exists $m>0$ such that $\left\|J\left(y_{n}\right)\right\|<m$ for each $n$. Hence $I(y) \leq$ $I_{g}(y) \leq \liminf I_{g}\left(y_{n}\right)=\liminf \left[I\left(y_{n}\right)+\varepsilon\left\|J\left(y_{n}\right)\right\|\right] \leq \liminf I\left(y_{n}\right)+m \varepsilon$ since $g$ is semi-regular positive semi-normal and hence $V$-convex.

The construction in Theorem 3.5 can be used to show that not only is $T$-convexity a necessary condition that $I$ be lower semi-continuous with respect to the convergence of that theorem, but also with respect to the convergence of Corollary 6.5.

The gap between the necessary and sufficient conditions for lower semi-continuity can now be described by the fact that $f$ can be $T$-convex without being continuous (but see the paragraph preceding Corollary 7.3).

Since $\Rightarrow$ is stronger than $\rightarrow$, the following corollary is immediate.

Corollary 6.6. If $y_{n} \Rightarrow y$ in $\Re\left(y_{o}\right)$ then $I(y) \leq \liminf I\left(y_{n}\right)$. 
7. We conclude with an existence theorem and some minor generalizations.

THEOREM 7.1. Let $f \in C(A \times Y)$ be nonnegative and $f_{\theta}$ be convex for each $\theta \in A$. If $\mathscr{N}\left(y_{o}\right)$ is $\Rightarrow$ compact and if $\inf \left\{I(y) \mid y \in \mathfrak{N}\left(y_{o}\right)\right\}<\infty$ then I attains its minimum on $\Re\left(y_{o}\right)$.

This result follows from Corollary 6.6.

COROLlary 7.2. Suppose that there exists $m>0$ such that for each $(\theta, s) \in A \times Y$ either

(i) There exists $M>0$ and $p>1$ such that $\|y\|_{\infty}<M$ and $f(\theta, s) \geq$ $m\|s\|^{p}$, or

(ii) $f(\theta, s) \geq m\|s\|^{q}$ where $q=2 k /(k+1)$. If $\inf \left\{I(y) \mid y \in \mathbb{R}\left(y_{o}\right)\right\}$ $<\infty$ then I attains its minimum on $\Re\left(y_{o}\right)$.

The corollary follows from Theorem 4.8.

Let $Y_{1}$ be a compact convex subset of $Y$. If $y_{o} \in A C$ and if $J\left(y_{o}, x\right) \in$ $Y_{1}$ for almost all $x \in G$, then let

$$
\mathfrak{M}_{1}\left(y_{o}\right)=\mathfrak{\Re}\left(y_{o}\right) \cap\left\{y \mid J(y, x) \in Y_{1} \text { for almost all } x \in G\right\} .
$$

Let $f \in C\left(A \times Y_{1}\right)$. If $I$ is lower semicontinuous on $\mathfrak{T}_{1}\left(y_{o}\right)$ then, as before, $f$ must be $T$-convex, i.e., there exists $g_{\theta}: Y_{1} \rightarrow \mathbf{R}$ where $g_{\theta}$ is convex and extends $f_{\theta}$ for each $\theta \in A$. Since $Y_{1}$ is compact, it follows that $g$ is continuous so, for this case, a necessary and sufficient condition that $I$ be lower semicontinuous is that $f$ be $T$-convex. Thus the next corollary follows from the preceding one.

COROLlaRY 7.3. Let $Y_{1}$ be a compact convex subset of $Y$ and $f \in$ $C\left(A \times Y_{1}\right)$ be T-convex. If, in addition, $f$ satisfies (i) or (ii) and $\inf \left\{I(y) \mid y \in \Re_{1}\left(y_{o}\right)\right\}<\infty$ then I attains its minimum on $\Re_{1}\left(y_{o}\right)$.

Let $Y_{2}$ be a compact subset of $Y$ and $f \in C\left(A \times Y_{2}\right)$. Let $Y_{1}$ be the convex hull of $Y_{2}$ and let $g$ be defined on $A \times Y_{1}$ by

$$
\begin{aligned}
& g(\theta, q)=\inf \left\{\sum_{i=1}^{n} \lambda_{i} f\left(\theta, p_{i}\right) \mid p_{i} \in Y_{2},\right. \\
&\left.\lambda_{i}>0, \sum \lambda_{i}=1, \text { and } \sum \lambda_{i} p_{i}=q\right\} .
\end{aligned}
$$

If $g \in C\left(A \times Y_{1}\right)$ is $T$-convex and if

$$
\inf \left\{I_{g}(y) \mid y \in \mathfrak{K}_{1}\left(y_{o}\right)\right\}<\infty,
$$


where $I_{g}(y)=\int_{G} g\left(y_{*}(x), J(y, x)\right) d x$, then, by Corollary 7.3, there exists $z \in \mathfrak{M}_{1}\left(y_{o}\right)$ such that $g(z)=\min \left\{I_{g}(y) \mid y \in \mathfrak{N}_{1}\left(y_{o}\right)\right\}$. Then $z$ is called a relaxed minimizer for $f$ on $Y_{2}$.

\section{REFERENCES}

1. J. M. Ball, Convexity conditions and existence theorems in nonlinear elasticity, Arch. Rational Mech. Anal., 63 (1977), 337-403.

2. Lamberto Cesari, An existence theorem of calculus of variations on parametric surfaces, Amer. J. Math., 74 (1952), 265-295.

3.___ Surface Area, Princeton Press, 1956.

4. A necessary and sufficient condition for lower semicontinuity, Bull. Amer. Math. Soc., 80 (1974), 467-472.

5. C. B. Morrey, Jr., Quasi-convexity and the lower semicontinuity of multiple integrals, Pacific J. Math., 2 (1952), 25-53.

6. __ Multiple Integrals in the Calculus of Variations, Springer Verlag Vol. 130, 1966. 7. Edward Silverman, $A$ sufficient condition for the lower semicontinuity of parametric integrals, Trans. Amer. Math. Soc., 167 (1972), 465-469.

$8 . \quad$, Lower semicontinuity of parametric integrals, Trans. Amer. Math. Soc., 175 (1973), 499-508.

$9 . \quad$, Strong quasi-convexity, Pacific J. Math., 46 (1973), 549-554.

10. L. H. Turner, The direct method in the calculus of variations, Purdue Thesis, 1957.

Received December 12, 1980.

PURDUE UNIVERSITY

WeST LAFAYETTE, IN 47907 